\title{
The Postprandial Effects of a Moderately High-Fat Meal on Lipid Profiles and Vascular Inflammation in Alzheimer's Disease Patients: A Pilot Study
}

Robin Altman ${ }^{1}$, Alison H. Keenan ${ }^{2}$, John W. Newman ${ }^{3}$ and John C. Rutledge ${ }^{1^{*}}$

${ }^{1}$ Division of Cardiovascular Medicine, Department of Internal Medicine, School of Medicine, University of California at Davis, Davis, CA 95616, California, USA

${ }^{2}$ Department of Nutrition, University of California at Davis, Davis, CA 95616, California, USA

${ }^{3}$ USDA ARS Western Human Nutrition Research Center, University of California at Davis, Davis, CA 95616, California, USA

*Corresponding author: John Rutledge, Department of Internal Medicine, Division of Cardiovascular Medicine Genome and Biomedical Sciences Facility, Ste. 5404451 East Health Sciences Drive University of California, Davis, CA 95616, California, USA, Tel: (530) 752-2182; E-mail: jcrutledge@ucdavis.edu

Received date: October 14, 2014; Accepted date: November 17, 2014; Published date: November 27, 2014

Copyright: (c) 2014 Altman R, et al. This is an open-access article distributed under the terms of the Creative Commons Attribution License, which permits unrestricted use, distribution, and reproduction in any medium, provided the original author and source are credited.

\begin{abstract}
Objective: Alzheimer's disease (AD) is a neurodegenerative disease of aging with unknown causative factors. Accumulating evidence suggests that inflammation and neurovascular dysfunction play important roles in AD. The postprandial period following a moderately high-fat meal is associated with vascular inflammation in young, healthy individuals; however, this relationship has not been investigated in Alzheimer's patients despite their exaggerated inflammatory state.
\end{abstract}

Methods: Patients with AD and age-matched control subjects were recruited through the UC Davis Alzheimer's Disease Center. All subjects consumed a moderately high-fat breakfast meal. Fasting and postprandial blood samples were collected for lipid, lipoprotein, and oxylipin analyses, as well as assays for cytokine levels and monocyte activation.

Results: The plasma lipid analyses revealed similar levels of triglycerides and esterified oxylipins between groups, but there was an interaction between postprandial non-esterified fatty acid (NEFA) levels and body mass index in the $A D$ group compared to the control subjects. The $A D$ group also had increased behenic acid and decreased linoleic and oleic acids in the postprandial period; however, these were not significantly different. Inflammatory assays revealed elevated fasting levels of interleukin (IL)-10 and IL-12 p70, but no change in monocyte activation in the AD group.

Conclusion: The postprandial period following a moderately high-fat meal is not associated with an exaggerated inflammatory state in Alzheimer's patients, and basal esterified oxylipin profiles do not indicate elevated oxidative stress. However, the baseline inflammatory state during fasting in AD patients includes elevated levels of plasma $\mathrm{IL}-10$ and IL-12 p70, which may indicate a balance between immune responses mediated by these interleukins.

Keywords: Alzheimer's disease; Postprandial; Lipid; Oxylipin; Cytokine; Monocyte activation; Vascular inflammation

\section{Introduction}

Alzheimer's disease is the most common form of dementia and a rapidly growing health concern worldwide. A growing body of evidence suggests Alzheimer's disease (AD) is a complex neurological disorder of aging that is influenced by a host of genetic and environmental factors. Epidemiological studies indicate that a high-fat diet may be one of these risk factors, and many studies have investigated the effects of long-term dietary interventions on the prevention of $\mathrm{AD}$ [1-6]. The acute postprandial effects of a moderately high-fat meal on potential risk factors for $\mathrm{AD}$, such as the blood lipid profile and vascular inflammation, have been less well-studied.

Alzheimer's disease is associated with an exacerbated inflammatory state in the brain [7-11], and it has been suggested peripheral vascular inflammation may contribute to the disease. Previous work has shown that acute systemic inflammation appears to exacerbate symptoms of chronic neurodegenerative disease [12], and epidemiological studies demonstrate a possible association between serum inflammatory markers and risk of developing $\mathrm{AD}$ or cognitive impairment [13]. Additionally, $\mathrm{AD}$ has been strongly linked to the metabolic syndrome, which includes perturbations in the normal blood lipid profile such as elevated cholesterol and triglycerides [14-17]. Although controversy still surrounds the role of triglyceride-rich lipoproteins in $\mathrm{AD}$, most evidence points toward a relationship between elevated plasma triglyceride levels and Alzheimer's disease [14-18].

In young, healthy individuals, increased vascular inflammation during the postprandial period following a moderately high-fat meal correlates with the peak increase in plasma triglycerides [19-21], and non-esterified fatty acids (NEFAs) serve as important mediators of this inflammatory effect $[22,23]$. Postprandial vascular inflammation also includes increased platelet and monocyte activation and aggregation, and elevated levels of soluble inflammatory markers such as IL- $1 \beta$ and TNF- $\alpha$ [19]. Additionally, elevated plasma esterified oxylipins indicate inflammatory oxidative stress processes, and may also play an important role in the development and progression of Alzheimer's disease [24-26]. 
Inflammation of the cerebrovasculature due to elevated blood lipid levels during the postprandial period may promote neurovascular disease, suggesting a potential mechanism contributing to the brain injury characteristic of $\mathrm{AD}$. The goal of this pilot study was to help elucidate whether the postprandial response following a moderately high-fat meal is associated with a perturbed lipid profile and exaggerated vascular inflammation in Alzheimer's patients.

\section{Materials and Methods}

\section{Materials}

FACS Lysing Solution, FACS Permeabilizing Solution, and fluorescently-labeled antibodies were obtained from BD Biosciences (San Jose, CA). All Vacutainer blood collection tubes were purchased from BD (Franklin Lakes, NJ). Brefeldin A, sodium azide, and bovine serum albumin (BSA) were obtained from Sigma (St. Louis, MO). Paraformaldehyde was obtained from Polysciences, Inc. (Warrington, PA).

\section{Subjects}

The study protocol was approved by the Institutional Review Board of the University of California, Davis. Seven subjects with probable AD and nine cognitively normal control subjects were recruited through the UC Davis Alzheimer's Disease Center cohorts. Written informed consent was obtained for all subjects using a consent document and procedure approved by the UC Davis Institutional Review Board. A Capacity Assessment Record was completed for each AD subject to ensure their ability to provide informed consent, and if an individual was not able to provide their own consent, a surrogate consent procedure (including written consent document) was also approved for use by the Institutional Review Board.

All subjects underwent a thorough clinical assessment through the UC Davis Alzheimer's Center prior to enrollment in the study. The diagnosis of dementia was based on DSM-IV criteria 6, and the diagnosis of probable $\mathrm{AD}$ was based on NINCDS-ADRDA criteria. Severity of dementia was determined by the Folstein Mini Mental State Exam (MMSE) [27], and Alzheimer's patients with a score of 16-26 (inclusive), indicative of mild to moderate dementia, were eligible to participate in the study. Subjects did not have evidence of any of the following: dementia due to another cause, seizure disorder, acquired immune deficiency syndrome, drug or alcohol abuse, current or past (within the past two years) myocardial infarction or congestive heart failure, clinically significant or uncontrolled medical condition, or use of an investigational drug before or during the study. Prior to the study day, subjects discontinued their use of lipid lowering drugs (for thirty days) and aspirin (for 14 days), where applicable. Subjects were matched between groups based on age and body mass index (BMI).

\section{Anthropometric measurements}

Body weight and height were measured on the day of the study by trained personnel using standard practices. BMI was calculated using the standard equation: (body mass in kilograms)/(height in meters $\left.)^{2}\right)$.

\section{Food diary and food frequency questionnaire}

For 72 hours prior to the study day, subjects were asked to complete a food diary that was provided by the study personnel. Subjects recorded food and beverage intake, vitamin and supplement intake, and duration/type of physical activity. For those subjects who were unable to independently complete the food diary, family members and/or caretakers assisted with completion of the diary. On the study day, subjects were asked to complete the Block Brief 2000 Food Frequency Questionnaire (FFQ) (NutritionQuest, Berkeley, CA). The FFQ was completed with the assistance of trained study personnel or a registered research dietician, and when possible, a family member and/or caretaker provided collateral history to help complete the FFQ. Information obtained from the food diaries and FFQs was analyzed to establish the baseline nutritive status of the study subjects, and to identify any abnormalities in their dietary behaviors that could have adversely affected the experimental outcomes. There were no indications that the baseline dietary habits of the subjects would have significantly affected the outcomes of the study.

\section{Study protocol and blood collection}

Prior to the study day, subjects fasted overnight for 12 hours. They were instructed to take their normal medications and to drink plenty of water. The study was performed at the Clinical and Translational Science Center Clinical Research Center (CCRC) located at the VA Hospital in Mather, CA. On the study day, subjects arrived at the CCRC in the morning and were fitted with intravenous saline locks to obtain blood samples during the study. A fasting blood sample was collected, and then subjects were fed a standardized $40 \%$ saturated fat breakfast meal (Table 1). Following consumption of the breakfast meal, subjects were asked to rest quietly for the duration of the study day. Subjects were provided with ad libitum access to water, but were not allowed to consume any other foods or caloric beverages for the remainder of the study. Postprandial blood samples were obtained at $1.5,3.5$, and 6 hours after the breakfast meal. Blood samples were immediately placed on ice and processed within one hour for plasma isolation or flow cytometric studies. To isolate plasma, blood samples were drawn into $\mathrm{K}_{2}$ EDTA Vacutainer tubes and centrifuged at 3,000 rpm for 15 minutes at $4^{\circ} \mathrm{C}$. The plasma fraction was immediately frozen at $-80^{\circ} \mathrm{C}$ until further analysis.

\begin{tabular}{|c|c|c|c|c|c|}
\hline Food Item & Amount(g & Energy(kcal) & Protein $(g)$ & $\begin{array}{l}\text { Carbohy } \\
\text { drate }(g)\end{array}$ & Fat(g \\
\hline $\begin{array}{lll}\begin{array}{l}\text { Plain } \\
\text { small }\end{array} & \text { Bagel, } & 1\end{array}$ & 75.0 & 206.3 & 7.9 & 40.1 & 1.2 \\
\hline $\begin{array}{l}\text { Cream Cheese, } 4 \\
\text { tsp }\end{array}$ & 19.3 & 67.5 & 1.5 & 0.5 & 6.7 \\
\hline Egg, 1 large & 50.0 & 74.5 & 6.2 & 0.6 & 5.0 \\
\hline Margarine, 2 tsp & 9.4 & 67.6 & 0.1 & 0.1 & 7.6 \\
\hline Cantaloupe, 1 cup & 226.8 & 79.4 & 2.0 & 19.0 & 0.6 \\
\hline Whole Milk, 1 cup & 244.0 & 148.8 & 8.0 & 11.4 & 8.2 \\
\hline Total & 624.5 & 644.0 & 25.7 & 71.6 & 29.3 \\
\hline$\%$ of energy & & & 15.7 & 43.9 & 40.4 \\
\hline
\end{tabular}

Table 1: Composition of the standardized breakfast meal containing approximately $40 \%$ of total energy derived from fat. 


\section{Lipid analyses}

Triglycerides and Cholesterol: Frozen/thawed plasma samples were used for all lipid analyses. Triglycerides, total cholesterol, and direct HDL cholesterol were determined colorimetrically after enzymatic hydrolysis and oxidation on a Poly-Chem instrument (PolyMedCo, Cortlandt Manor, NY) using the indicator quinoneimine. The Friedewald formula was used to calculate LDL cholesterol [28]: LDL cholesterol=total cholesterol-[HDL cholesterol+(triglyceride/5)].

Non-Esterified Fatty Acids: The non-esterified fatty acid (NEFA) concentrations of the plasma samples were measured in duplicate using an enzymatic colorimetric assay kit (Wako Diagnostics, Richmond, VA) according to the manufacturer's instructions.

Oxylipin Analysis: The majority of circulating oxylipins are esterified into lipoprotein particles [29], and their release at the vascular endothelium by lipoprotein lipase may participate in inflammatory signaling [22]. Therefore sample extracts were subjected to alkaline hydrolysis prior to analysis to release this bound lipid pool, and a suite of arachidonic, eicosapentaenoic and docosahexaenonic acid metabolites were measured.

Frozen/thawed plasma samples were hydrolyzed and extracted by solid phase extraction (SPE) using $3 \mathrm{cc}, 60 \mathrm{mg}$ Oasis HLB columns (Waters, Milford, MA). Samples were enriched with an antioxidant solution of butylated hydroxyl toluene and the divalent cation chelator EDTA. Once eluted, samples were enriched with $\sim 1 \mathrm{mg}$ glycerol and residual solvent was removed under vacuum. Glycerol plugs were stored at $80^{\circ} \mathrm{C}$ until reconstitution in methanol-containing $100 \mathrm{nM}$ of the internal standards 1-cyclohexyluriedo-3-dodecanoic acid (CUDA) and 1-phenyluriedo-3-hexanoic acid (PHAU).

Oxylipins and deuterated surrogates were analyzed by ultraperformance liquid chromatography-tandem mass spectrometry (UPLC-MS/MS) as previously described [30,31]. Briefly, analytes were separated on a $2.1 \times 150 \mathrm{~mm}, 1.7 \mu \mathrm{m}$ Acquity $\mathrm{BEH}$ column at $60^{\circ} \mathrm{C}$ on a Waters Acquity UPLC. Analytes were ionized in negative mode by electrospray ionization and data were acquired and collected in multireaction monitoring mode with an ABI 4000QTRAP triple quad mass spectrometer. Surrogate recoveries of reported analytes were acceptable.

\section{Metabolite analysis}

Plasma samples for metabolomics assays were stored at $-80^{\circ} \mathrm{C}$ and underwent two freeze-thaw cycles prior to extraction. The samples were extracted and derivatized as described previously [32]. The derivatized samples $(0.5 \mu \mathrm{L})$ were injected onto an Agilent 6890 gas chromatograph (Santa Clara, CA) at $50^{\circ} \mathrm{C}$ (ramped to $250^{\circ} \mathrm{C}$ ) in splitless mode with 25 seconds of splitless time. The chromatograph was equipped with a $30 \mathrm{mx} 0.25 \mathrm{~mm}$ i.d.x0.25 $\mu \mathrm{m}$ Rtx5Sil-MS column with a $10 \mathrm{~m}$ integrated guard column (Restek, Bellefonte, PA). The chromatography conditions were as follows: constant flow rate of 1 $\mathrm{mL} / \mathrm{min}$, oven temperature ramped from $50^{\circ} \mathrm{C}$ to $330^{\circ} \mathrm{C}$, and total run time of 22 minutes. Mass spectrometry was accomplished by a Leco Pegasus IV time of flight mass spectrometer (St. Joseph, MI) under the following conditions: transfer line temperature of $280^{\circ} \mathrm{C}$, electron ionization at $-70 \mathrm{eV}$, and ion source temperature of $250^{\circ} \mathrm{C}$. Mass spectra were acquired from m/z 85-500 at 17 spectra s- 1 and $1850 \mathrm{~V}$ detector voltage. All results were exported to the Fiehn Lab servers and processed by the metabolomics BinBase database [33,34]. All entries to the database were matched against both the Fiehn Lab mass spectral library of metabolites and the NIST05 commercial library using retention index and mass spectrum information. Quantifier ions were identified for each metabolite based on the information in BinBase. The quality control mixtures (containing 30 metabolites) established external 5-point calibration curves to control for instrument sensitivity. Furthermore, each individual chromatogram was controlled against the total number of identified metabolites and the total peak intensities in order to prevent outliers from confusing the statistical analysis. The differences in mean plasma metabolite concentrations between groups were evaluated using false discovery rate corrections.

\section{Cysteine and homocysteine measurements}

Plasma homocysteine and cysteine were determined by highpressure liquid chromatography with post-column fluorescence detection as described previously [35].

\section{Flow cytometry}

Whole blood was drawn into sodium heparin Vacutainer tubes, immediately placed on ice, and aliquoted within 30 minutes for flow cytometry staining. An aliquot of whole blood was incubated with Brefeldin A (0.002\%) for four hours at $37^{\circ} \mathrm{C}$ in a $95 \%$ air $/ 5 \% \mathrm{CO} 2$ incubator. Subsequent steps were performed at room temperature in the dark. Following the incubation period, small aliquots of the whole blood were stained with FITC-labeled mouse anti-CD14 antibody (catalog \# 347493) for 20 minutes. Samples were lysed with FACS Lysing Solution, vortexed gently, and incubated for 10 minutes. Following centrifugation to collect the cells, samples were permeabilized using FACS Permeabilizing Solution for 10 minutes. Samples were washed once with wash buffer (PBS containing $0.5 \%$ BSA and $0.1 \%$ sodium azide) and then centrifuged to collect the cells. APC-labeled mouse anti-TNF- $\alpha$ (catalog \# 340534) and PE-labeled mouse anti-IL-1 $\beta$ (catalog \# 340516) antibodies were added to the samples and left to incubate for 30 minutes, after which they were washed once with wash buffer and fixed in $1 \%$ paraformaldehyde. Samples were run on a FACScan Flow Cytometer (BD Biosciences, San Jose, CA) and analyzed using FlowJo software (Tree Star, Ashland, OR). The monocyte population was determined by the light scatter characteristics of the cells, as well as the extracellular expression of CD14, a monocyte marker.

\section{Multiplex plasma cytokine analysis}

Levels of plasma cytokines were measured using the Human ProInflammatory 7-Plex Assay Ultra-Sensitive Kit from Meso Scale Discovery (Gaithersburg, Maryland) according to the manufacturer's instructions. The following cytokines were analyzed in duplicate: interleukin (IL)-1 $\beta$, IL-12 p70, interferon- $\gamma$ (IFN- $\gamma$ ), IL-6, IL-8, IL-10, and tumor necrosis factor (TNF)- $\alpha$.

\section{Statistical analyses}

All values are expressed as means \pm standard deviation, unless otherwise noted. Normally-distributed data were analyzed using SigmaPlot software (San Jose, CA), and statistical significance was determined using two-way repeated measures ANOVA followed by the Holm-Sidak method for multiple comparisons testing, unless otherwise noted. A p-value of less than 0.05 was considered significant. 
Citation: $\quad$ Altman R, Keenan AH, Newman JW, Rutledge JC (2014) The Postprandial Effects of a Moderately High-Fat Meal on Lipid Profiles and

\section{Results}

\section{Cholesterol and triglyceride levels are not different between $\mathrm{AD}$ and control groups during the fasting or postprandial states}

Descriptions of the two study groups (Alzheimer's patients and control subjects), including mean values for age, MMSE score, height, weight, and BMI are shown in Table 2. The differences between the two groups did not reach statistical significance for any of these parameters.

\begin{tabular}{|l|l|l|}
\hline & AD Patients & Control Subjects \\
\hline No. of individuals (M/F) & $7(3 / 4)$ & $9(3 / 6)$ \\
\hline MMSE & $22.7 \pm 2.4$ & $>26$ \\
\hline Age, years & $78 \pm 9.3$ & $76 \pm 6.8$ \\
\hline Height, cm & $162.7 \pm 9.44$ & $165.9 \pm 8.13$ \\
\hline Weight, $\mathrm{kg}$ & $66.2 \pm 8.14$ & $86.0 \pm 32.7$ \\
\hline BMl, kg/m2 & $25.0 \pm 2.00$ & $30.6 \pm 9.34$ \\
\hline Total cholesterol, mg/dL & $222.6 \pm 48.3$ & $220.7 \pm 42.3$ \\
\hline HDL, mg/dL & $61.9 \pm 16.7$ & $54.8 \pm 15.1$ \\
\hline LDL, $\mathrm{md} / \mathrm{dL}$ & $140.8 \pm 27.8$ & $127.0 \pm 37.5$ \\
\hline
\end{tabular}

MMSE=Mini-Mental State Exam [27]; HDL = high density lipoprotein; LDL = low density lipoprotein. Clinical lipid measurements were obtained during fasting. Data are expressed as means $\pm S D$. The differences between the two groups did not reach statistical significance $(p<0.05)$

Table 2: Characteristics of Alzheimer's patients and control subjects enrolled in the study.

One of our primary objectives was to characterize the postprandial lipid and lipoprotein response to a moderately high-fat meal challenge in $\mathrm{AD}$ patients compared to control subjects. To establish baseline values for the two groups, we first determined that fasting plasma cholesterol levels, including HDL, LDL, and total cholesterol, were not different in $\mathrm{AD}$ patients compared to control subjects (Table 2). As shown in Figure 1, plasma triglyceride levels were slightly higher in the control group compared to the $\mathrm{AD}$ group during fasting and the postprandial period, but these differences did not reach statistical significance. In agreement with previous studies [19,36], triglyceride levels increased from fasting to a postprandial peak at 3.5 hours, and approached baseline values at 6 hours.

\section{The relationship between postprandial NEFA and BMI differs between $\mathrm{AD}$ and control groups}

As it is thought NEFAs help mediate postprandial inflammation, we investigated plasma concentrations of these lipids [22,23]. No differences in plasma NEFA were detected between the $\mathrm{AD}$ and control groups at any time point. The same trend was observed in each group, where NEFA concentration dropped from fasting during the postprandial period (through 3.5 hours) and approached baseline values at the 6-hour time point. When expressed as a function of BMI, however, the slopes of the linear relationship between BMI and NEFA levels were significantly different between the two groups at the 3.5- hour time point (Figure 2). This suggests that postprandial NEFA excursions are greater in $\mathrm{AD}$ patients relative to $\mathrm{BMI}$-matched controls at 3.5 hours after the consumption of a moderately high-fat meal.

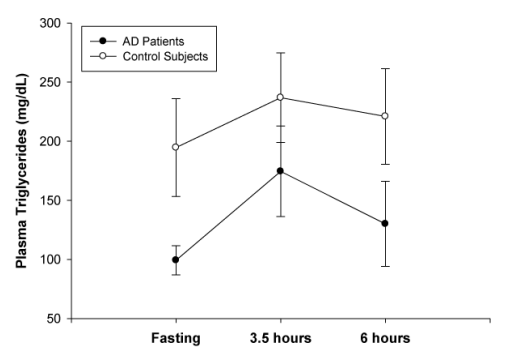

Figure 1: Plasma triglyceride levels in Alzheimer's (AD) patients and control subjects. Whole blood samples were obtained at fasting, and at 3.5 and 6 hours following consumption of a moderately high-fat meal. The level of plasma triglycerides was not significantly different between the AD group $(n=7)$ and the control group $(n=9)$ at any time point. Both groups had increased levels of triglycerides at 3.5 hours postprandially, with this difference reaching significance in the AD group. Data are expressed as means \pm SEM. ${ }^{*} p<0.05$ compared to fasting (two-way repeated measures ANOVA).

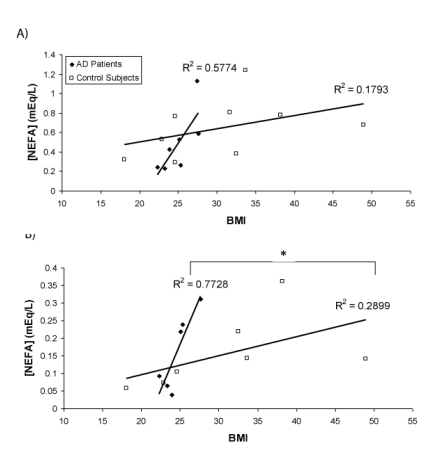

Figure 2: The relationship between non-esterified fatty acid (NEFA) levels and body mass index (BMI) differs between Alzheimer's (AD) patients and control subjects. Plasma samples were collected during fasting (A) or 3.5 hours following consumption of a moderately high-fat meal (B). NEFA levels are expressed as a function of body mass index (BMI) for each subject. The slope of the relationship between plasma NEFA levels and BMI is significantly steeper in the AD group $(n=6)$ compared to the control group $(n=7)$ at the 3.5 hour time point $\left({ }^{*} p<0.05\right.$, ANCOVA). The linear relationship between plasma NEFA levels and BMI for each group is shown as a solid dark line, with the corresponding R2 value noted to describe the strength of the relationship.

\section{Esterified eicosanoids and docosahexanoids do not differ between $\mathrm{AD}$ and control groups}

We then investigated the levels of plasma esterified oxylipins as an indication of oxidative stress $[25,26]$. Analytical performance was within acceptable limits for all reported analytes and the variance was 
Page 5 of 9

equivalent between the two study groups. As shown in Figure 3, no significant differences were detected between control subjects and those with Alzheimer's disease. Notably, the putative oxidative stress marker 9-HETE [37] was not significantly different between the subject groups, and isoprostanes were not routinely detected in these samples.

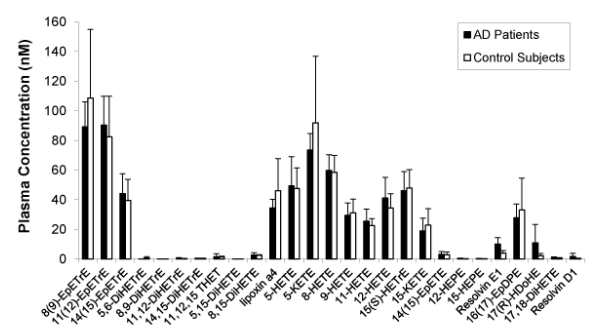

Figure 3: Oxylipin composition of plasma samples from Alzheimer's (AD) patients and control subjects. Whole blood samples were obtained at fasting, after which the plasma fractions were isolated and analyzed by liquid chromatography-tandem mass spectrometry for the following analytes: 8(9)-epoxy-8Z, 11Z, 14Zeicosatrienoic acid (EpETrE), 11(12)-EpETrE, 14(15)-EpETrE, 5,6dihydroxy- 5Z, 8Z, 11Z- eicosatrienoic acid (DiHETrE), 8,9DiHETrE, 11,12-DiHETrE, 14,15-DiHETrE, 11,12,15 trihydroxyeicosatrienoic acid (THET), 5,15-dihydroxy-5Z,9E, 11Z, 13E- eicosatetraenoic acid (DiHETE), 8,15-DiHETE, lipoxin a4, 5hydroxyeicosatetraenoic acid (HETE), 5- 5- oxo- 6E, 8Z, 11Z, 14Zeicosatetraenoic acid (KETE), 8-HETE, 9-HETE, 11-HETE, 12HETE, 15(S)-hydroxy-8Z,11Z,13E-eicosatrienoic acid (HETrE), 15KETE, 14(15)-epoxy- 5Z, 8Z, 11Z, 17Z- eicosatetraenoic acid (EpETE), 12-hydroxy-5Z, 8Z, 10E, 14Z, 17Z- eicosapentaenoic acid (HEPE), 15-HEPE, Resolvin E1, 16(17)-epoxy-4Z,7Z,10Z,13Z,19Zdocosapentaenoic acid (EpDPE), 17(R)-hydroxy- 4Z, 7Z, 10Z, 13Z, 15E, 19Z-docosahexaenoic acid (HDoHE), 17,18-dihydroxy-5Z,8Z, $11 Z, 14 Z$ - eicosatetraenoic acid (DiHETE), and Resolvin D1. Data are expressed as means $\pm \mathrm{SD}(\mathrm{n}=5 \mathrm{AD}$ patients, and 5 control subjects).

\section{Metabolomics analyses did not identify AD-specific changes in postprandial NEFA profiles}

A metabolomics screen of plasma was performed to provide a broad assessment of metabolic changes in the cohorts. Of the 365 analytes observed in the screen, four in the fasting state and five in the postprandial state were different between groups; however, these differences did not reach statistical significance after controlling for false discovery rate (Table 3 ). The postprandial condition revealed elevated levels of behenic acid, cystine, and taurine, and reduced levels of linoleic and oleic acids in the Alzheimer's group. During fasting, the AD patients had decreased levels of phosphoric acid and glyoxalurea, and elevated levels of fructose.

\begin{tabular}{|c|c|c|c|}
\hline & $A D$ & Control & $P$-value \\
\hline \multicolumn{4}{|l|}{ Postprandial } \\
\hline Behenic (docosanoic) acid & $959 \pm 116$ & $759 \pm 234$ & 0.046 \\
\hline Linoleic acid & $619 \pm 218$ & $925 \pm 273$ & 0.026 \\
\hline
\end{tabular}

\begin{tabular}{|l|l|l|l|}
\hline Oleic acid & $1597 \pm 683$ & $2577 \pm 1124$ & 0.05 \\
\hline Cystine & $14343 \pm 2768$ & $10214 \pm 4575$ & 0.043 \\
\hline Taurine & $846 \pm 328$ & $461 \pm 362$ & 0.044 \\
\hline Fasting & $74528 \pm 8966$ & $88939 \pm 8020$ & 0.0058 \\
\hline Phosphoric acid & $1555 \pm 281$ & $1900 \pm 254$ & 0.025 \\
\hline Glyoxalurea & $2215 \pm 670$ & $1471 \pm 408$ & 0.029 \\
\hline Fructose & & &
\end{tabular}

Table 3: Metabolites displaying trends toward different plasma concentrations in Alzheimer's patients (AD) and control subjects .

${ }^{*}$ Data are expressed as means $\pm \mathrm{SD}$. $P$-values determined by t-test and reported for those metabolites with $p \leq 0.05$ compared to control. After controlling for false discovery rate, none of the metabolites were shown to be significantly different between groups.

\section{No difference in fasting or postprandial homocysteine or cysteine levels between $\mathrm{AD}$ and control groups}

As our initial metabolomics screen identified increased postprandial levels of the sulfur-containing amino acids cystine and taurine in the $\mathrm{AD}$ group, and previous studies have demonstrated a correlation between elevated plasma homocysteine (Hcy) levels and presence of Alzheimer's disease [38-40], we further investigated plasma cysteine and Hcy levels. We conducted additional liquid chromatography measurements and demonstrated no changes in cysteine or Hcy levels between the two groups at any time point.

\section{Elevated levels of TNF- $\alpha$-positive monocytes are present during fasting in AD patients, with no difference in IL-1 $\beta$ - positive monocytes between groups}

To investigate levels of vascular inflammation, we used flow cytometric analysis to determine the percent of monocytes expressing high levels of intracellular TNF- $\alpha$ or IL- $1 \beta$. In the AD group, there was a higher percent of TNF- $\alpha$-positive monocytes compared to the control group at the fasting, 1.5 hour, and 3.5 hour time points (Figure 4A). This increase was particularly prominent at the fasting time point, where the TNF- $\alpha$-positive monocytes measured $35.9 \%$ in the $\mathrm{AD}$ group compared to $25.2 \%$ in the control group. However, this difference did not reach statistical significance. The percent of IL-1 $\beta$ positive monocytes increased during the postprandial period, from fasting levels to a postprandial peak at 3.5 hours (Figure $4 \mathrm{~B}$ ). There was no difference in the percent of monocytes expressing IL- $\beta$ between the $\mathrm{AD}$ and control groups at any time point.

\section{Increased levels of IL-10 and IL-12 p70 characterize the fasting state in $\mathrm{AD}$ patients}

We further sought to compare the vascular inflammatory state characteristic of the postprandial period between Alzheimer's patients and control subjects through the multiplex analysis of a panel of cytokines classically related to inflammation. We assayed fasting and postprandial plasma samples for the following cytokines: interleukin (IL)-1 $\beta$, IL-12 p70, interferon- $\gamma$ (IFN- $\gamma$ ), IL-6, IL-8, IL-10, and tumor necrosis factor (TNF)- $\alpha$. Our analysis revealed differences in IL-10 and IL-12 p70 between the AD and control groups. As seen in Figure 5, 
levels of both cytokines were increased in the Alzheimer's patients during fasting compared to the control group, while IL-12 p70 was also elevated in the fasting state compared to the postprandial period in the $\mathrm{AD}$ group. The control group did not demonstrate a similar trend between time points.

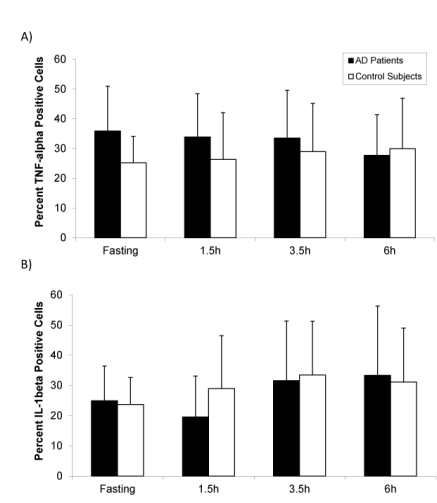

Figure 4: The percent of cluster of differentiation 14 (CD14)positive monocytes expressing either intracellular tumor necrosis factor (TNF)- $\alpha$ (A) or intracellular interleukin (IL)-1 $\beta$ (B) in Alzheimer's (AD) patients and control subjects. Whole blood samples were obtained from subjects during fasting, and then 1.5 , 3.5 , and 6 hours after consumption of a moderately high-fat meal and analyzed by flow cytometry. (A) There was a trend toward higher levels of TNF- $\alpha$-positive monocytes in the AD group $(n=6)$ compared to control $(n=7)$. (B) There was no difference in the percent of IL-1 $\beta$-positive monocytes between the $\mathrm{AD}(\mathrm{n}=7)$ and control groups $(n=8)$. Data are expressed as means $\pm S D$, and $\mathrm{p}<0.05$ was considered significant.

\section{Discussion}

Although previous studies have investigated the lipid profile and inflammatory markers in Alzheimer's disease patients compared to cognitively normal subjects, to our knowledge, this is the first analysis of these same factors during the postprandial state. We hypothesized that consumption of a moderately high-fat meal is associated with elevated levels of potentially injurious lipids and exaggerated vascular inflammation in the postprandial period in $\mathrm{AD}$ patients. We demonstrate here the postprandial period following a moderately high-fat meal is not associated with an exaggerated inflammatory state in $\mathrm{AD}$ patients with minimal cognitive impairment compared to agematched controls. However, we observed increased levels of activated monocytes, IL-10, and IL-12 p70 during fasting in AD patients. Our results also indicate the lipid profile of the $\mathrm{AD}$ group is characterized by reduced levels of linoleic and oleic acids, and an interaction between postprandial non-esterified fatty acid (NEFA) levels and body mass index.

It is increasingly recognized that inflammatory processes in the central nervous system characterize Alzheimer's disease and may mediate neurotoxicity [41]. Some lines of evidence also suggest systemic and/or peripheral vascular inflammation may contribute to Alzheimer's pathology [12,13]. Assessment of the fasting plasma oxylipin profiles in our study did not show evidence of a sustained elevation in lipoprotein oxidative damage in the AD patients, which would be consistent with a sustained oxidative stress, and we did not observe differences in 9-HETE, which has been reported as a marker of oxidative stress [37]. Furthermore, there were no significant differences in omega- 6 and omega- 3 polyunsaturated fatty acids, suggesting there was a similarity in background dietary lipids between the two groups [30]. However, these findings do not preclude changes in acute inflammatory reactions to a meal.

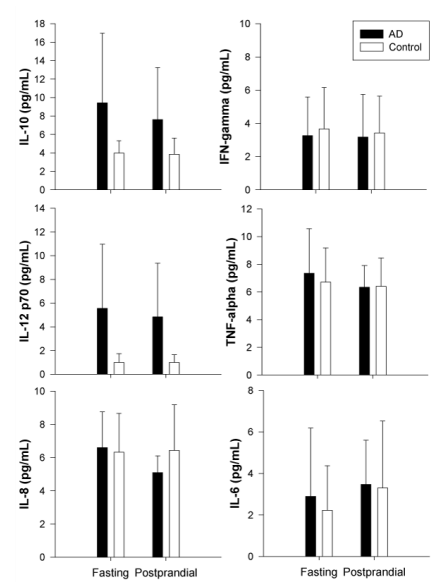

Figure 5: Plasma cytokine levels $(\mathrm{pg} / \mathrm{mL}$ ) in Alzheimer's patients (AD) and control subjects (Control). Whole blood was obtained during fasting and 3.5 hours after consumption of a moderately high-fat meal (postprandial). The following cytokines were measured in the plasma fraction: interleukin (IL)-1 $\beta$, IL-12 p70, interferon- $\gamma$ (IFN- $\gamma)$, IL-6, IL-8, IL-10, and tumor necrosis factor (TNF)- $\alpha$. IL-10 and IL12 p70 were elevated in AD patients during fasting, while IL-12 p70 was also elevated in AD patients in the postprandial period. Data are expressed as means $\pm \mathrm{SD}$. ${ }^{*} p<0.05$ compared to control, and ${ }^{\#} p<0.05$ for fasting $\mathrm{AD}$ compared to postprandial AD (two-way repeated measures ANOVA).

Of the inflammatory mediators investigated, we observed increased levels of IL-10 and IL-12 (p70 subunit) in the Alzheimer's patients during the fasting state, however these differences were not sustained during the postprandial period. IL-10 and IL-12 play important roles in the differentiation and regulation of helper $\mathrm{T}$ (Th) cells as part of the cell-mediated immune response. The type I (Th1) response primarily functions in an inflammatory capacity and involves IL-12, while type II (Th2) cells serve as a major source of IL-10 that functions in an anti-inflammatory capacity [42-44]. It has been suggested that $\mathrm{AD}$ may in part reflect deficiency of a protective Th2 response to combat Th1-mediated pathology [45]. Our observations of elevations in fasting IL-10 suggest that further investigation into the balance between Th1 and Th2 responses in AD could be beneficial. Our results contrast those of previous studies that were unable to detect these two cytokines or observed no difference between serum and cerebrospinal fluid from $\mathrm{AD}$ and control groups [46-49]. However, it is important to note the majority of these studies did not specify the fasted or fed state of the subjects. We observed differences in IL-10 and IL-12 during the fasting state, suggesting the fasting environment may be more sensitive to the changes we detected.

Prior work has revealed the potential involvement of peripheral blood mononuclear cells (PBMCs) in inflammation in AD. Elevated levels of PBMCs from Alzheimer's patients express pro-inflammatory TNF- $\alpha$ and cyclooxygenase (COX)-2, pro-apoptotic PARP-1, and the 
adhesion-related marker of activation, CD38. [11] We observed a nonsignificant trend towards a higher percentage of TNF- $\alpha$-positive monocytes in the Alzheimer's group at fasting consistent with these previous studies.

It is interesting to note that the greatest increase in TNF- $\alpha$-positive monocytes in the $\mathrm{AD}$ group coincided with the point at which total plasma non-esterified fatty acid (NEFA) levels were highest, i.e. the fasting state. This observation may potentially be indicative of higher vascular inflammatory activity at similar concentrations of NEFAs. Futhermore, we observed a correlation between NEFA levels and BMI that was significantly stronger in the Alzheimer's group 3.5 hours postprandially. Relative to their BMI, AD subjects had higher levels of NEFAs, which invites the question of whether they have a greater propensity for inflammatory activation. Further studies are needed to elucidate the underlying mechanism behind this observation and to account for potential effects of gender that might influence BMI, as well as adipose distribution and lipolytic behavior.

After subjects consumed a $40 \%$ saturated fat meal, we noted an increasing trend in the plasma levels of triglycerides from fasting to a postprandial peak at 3.5 hours. This correlates well with previous studies showing a similar peak in plasma triglycerides at about 3.5 hours after subjects consumed a moderately high-fat meal. [19] The postprandial increase in triglcyerides was more pronounced in the $\mathrm{AD}$ group than the control group. However, the control group showed consistently higher levels of plasma triglycerides at each time point (fasting, 3.5 hours and 6 hours postprandially,) which may help account for the blunted postprandial response. Measurements of plasma triglyceride levels in AD patients compared to control subjects have met with considerable variation among studies. Our observation is consistent with reports of decreased triglyceride levels in $\mathrm{AD}$ patients with a similar degree of dementia compared to our patients (as evidenced by the MMSE scores) [50].

To further investigate the distribution of specific fatty acids across time points between the two groups we conducted a global metabolomics analysis. This experiment revealed differences in the plasma concentrations of three fatty acids in the postprandial period: behenic acid levels were elevated, while oleic and linoleic acids were decreased in the $\mathrm{AD}$ group. These results correlate well with previous studies demonstrating reduced plasma and brain levels of oleic and linoleic acids in $\mathrm{AD}$ patients [51-53]. Whether the observed alterations in these two fatty acids reflect differences in intake or metabolism, or a combination of the two, presents an intriguing question. It would be beneficial to provide a controlled dietary regimen prior to the defined study breakfast meal to address this question. We further probed the sulfur-containing amino acids cysteine and homocysteine as part of our metabolomics analysis and did not observe any differences between the two study groups. However, this does not preclude the possibility that subtle changes in sulfur metabolism may be occurring in the postprandial period in $\mathrm{AD}$ subjects, and these changes may have direct relevance to the reported dysregulation of cysteine/ homocysteine in Alzheimer's disease [38-40,54].

The primary aspect of our study design warranting further consideration is the small number of subjects. However, other investigations focused on postprandial inflammatory responses following various dietary challenges used similarly small samples sizes [55-58]. In our cohort each subject served as their own control, which helped reduce variability among the different time points. For vulnerable populations, such as cognitively impaired Alzheimer's patients, the feasibility of recruiting large numbers of individuals is questionable. The primary aim of the studies presented here was to establish preliminary observations regarding the postprandial response to a moderately high-fat diet challenge in AD. Large cohorts of study subjects were beyond the scope of this pilot project and are planned for future work based on our initial pilot observations.

Finally, the evolution of $\mathrm{AD}$ ranges from the preclinical with no cognitive impairment to the vegetative state. The quantity and composition of food intake also varies considerably during this evolution, which would be expected to have effects on the postprandial inflammatory response. We have focused our study on those $\mathrm{AD}$ patients with minimal cognitive impairment with the hope that intervention at this early stage of the disease could attenuate the disease process. As biomarkers of $\mathrm{AD}$ improve, future studies should investigate the postprandial state in those persons at high risk for developing cognitive impairment.

In conclusion, we observed a combination of increased pro- and anti-inflammatory mediators, including IL-10, IL-12, and TNF- $\alpha$ positive monocytes in Alzheimer's patients during fasting, and a stronger interaction between postprandial NEFA levels and body mass index in the $\mathrm{AD}$ group compared to the control subjects. This may be further evidence of a heightened inflammatory state in AD. However, our results do not suggest that this inflammation is exacerbated in the postprandial state. We also identified differences in the metabolomics profile between $\mathrm{AD}$ and control subjects, which suggest potential targets for future investigations. Our work has clinical relevance in that it provides insight into potential baseline inflammatory mechanisms and metabolic differences that may characterize Alzheimer's disease.

\section{Acknowledgement}

The authors gratefully thank the following individuals for their generous assistance with this project: Theresa Tonjes of the Havel Lab Assay Services at the University of California, Davis performed all the cholesterol and triglyceride measurements for this study. Dr. Oliver Fiehn and the UC Davis Metabolomics Core performed the metabolomics analyses. Carol Oxford of the UC Davis Optical Biology Core Facility provided expert flow cytometry assistance. Dr. Xin Lin in the laboratory of Dr. Josh Miller performed the homocysteine and cysteine analyses. Dr. Danielle Harvey provided statistical analyses and consultation.

The authors also extend their gratitude to the following individuals at the UC Davis Alzheimer's Disease Center: Dr. Charles DeCarli, Esther Lara, Alexandra Butler, and Terry Bennett. In addition, this study would not have been possible without the generous individuals who volunteered as subjects, and the support of the staff and nursing personnel at the CCRC at the VA Hospital in Mather, CA.

\section{Funding Sources}

This work was supported by a pilot grant from the University of California, Davis Alzheimer's Disease Center Core (P30 AG10129-15) and NIH awards (AG039094, AG045541) to J.C.R., and by an American Heart Association Western States Affiliate fellowship to R.A.

This work was made possible by Grant Number UL1 RR024146 from the National Center for Research Resources (NCRR), a component of the National Institutes of Health (NIH), and NIH Roadmap for Medical Research. In addition, part of this investigation 
was conducted in a facility constructed with support from Research Facilities Improvement Program Grant Number C06 RR12088-01 from the NCRR, NIH. The contents of this work are solely the responsibility of the authors and do not necessarily represent the official view of NCRR or NIH.

\section{References}

1. Devore EE, Grodstein F, van Rooij FJ, Hofman A, Rosner B, et al. (2009) Dietary intake of fish and omega-3 fatty acids in relation to long-term dementia risk. Am J Clin Nutr 90: 170-176.

2. Gustaw-Rothenberg K (2009) Dietary patterns associated with Alzheimer's disease: population based study. Int J Environ Res Public Health 6: 1335-1340.

3. Kalmijn S, Launer LJ, Ott A, Witteman JC, Hofman A, et al. (1997) Dietary fat intake and the risk of incident dementia in the Rotterdam Study. Ann Neurol 42: 776-782.

4. Luchsinger JA, Tang MX, Shea S, Mayeux R (2002) Caloric intake and the risk of Alzheimer disease. Arch Neurol 59: 1258-1263.

5. Petot GJ, Friedland RP (2004) Lipids, diet and Alzheimer disease: an extended summary. J Neurol Sci 226: 31-33.

6. Scarmeas N, Luchsinger JA, Schupf N, Brickman AM, Cosentino S, et al. (2009) Physical activity, diet, and risk of Alzheimer disease. JAMA 302: 627-637.

7. Akiyama H, Barger S, Barnum S, Bradt B, Bauer J, et al. (2000) Inflammation and Alzheimer's disease. Neurobiol Aging 21: 383-421.

8. DeKosky ST, Ikonomovic MD, Wang X, Farlow M, Wisniewski S, et al. (2003) Plasma and cerebrospinal fluid alpha1-antichymotrypsin levels in Alzheimer's disease: correlation with cognitive impairment. Ann Neurol 53: 81-90.

9. Engelhart MJ, Geerlings MI, Meijer J, Kiliaan A, Ruitenberg A, et al. (2004) Inflammatory proteins in plasma and the risk of dementia: the rotterdam study. Arch Neurol 61: 668-672.

10. Kálmán J, Juhász A, Laird G, Dickens P, Járdánházy T, et al. (1997) Serum interleukin-6 levels correlate with the severity of dementia in Down syndrome and in Alzheimer's disease. Acta Neurol Scand 96: 236-240.

11. Kassner SS, Bonaterra GA, Kaiser E, Hildebrandt W, Metz J, et al. (2008) Novel systemic markers for patients with Alzheimer disease? - a pilot study. Curr Alzheimer Res 5: 358-366.

12. Cunningham C, Campion S, Lunnon K, Murray CL, Woods JF, et al. (2009) Systemic inflammation induces acute behavioral and cognitive changes and accelerates neurodegenerative disease. Biol Psychiatry 65: 304-312.

13. Rosenberg PB (2005) Clinical aspects of inflammation in Alzheimer's disease. Int Rev Psychiatry 17: 503-514.

14. Cankurtaran M, Yavuz BB, Halil M, Dagli N, Cankurtaran ES, et al. (2005) Are serum lipid and lipoprotein levels related to dementia? Arch Gerontol Geriatr 41: 31-39.

15. Raffaitin C, Gin H, Empana JP, Helmer C, Berr C, et al. (2009) Metabolic syndrome and risk for incident Alzheimer's disease or vascular dementia: the Three-City Study. Diabetes Care 32: 169-174.

16. Sabbagh M, Zahiri HR, Ceimo J, Cooper K, Gaul W, et al. (2004) Is there a characteristic lipid profile in Alzheimer's disease? J Alzheimers Dis 6: 585-589.

17. Razay G, Vreugdenhil A, Wilcock G (2007) The metabolic syndrome and Alzheimer disease. Arch Neurol 64: 93-96.

18. Burgess BL, McIsaac SA, Naus KE, Chan JY, Tansley GHK, et al. (2006) Elevated plasma triglyceride levels precede amyloid deposition in Alzheimer's disease mouse models with abundant A[beta] in plasma. Neurobiology of Disease 24: 114-127.

19. Hyson DA, Paglieroni TG, Wun T, Rutledge JC (2002) Postprandia lipemia is associated with platelet and monocyte activation and increased monocyte cytokine expression in normolipemic men. Clin Appl Thromb Hemost 8: 147-155.
20. van Oostrom AJ, Rabelink TJ, Verseyden C, Sijmonsma TP, Plokker HW, et al. (2004) Activation of leukocytes by postprandial lipemia in healthy volunteers. Atherosclerosis 177: 175-182.

21. Gill JM, Caslake MJ, McAllister C, Tsofliou F, Ferrell WR, et al. (2003) Effects of short-term detraining on postprandial metabolism, endothelial function, and inflammation in endurance-trained men: dissociation between changes in triglyceride metabolism and endothelial function. J Clin Endocrinol Metab 88: 4328-4335.

22. Wang L1, Gill R, Pedersen TL, Higgins LJ, Newman JW, et al. (2009) Triglyceride-rich lipoprotein lipolysis releases neutral and oxidized FFAs that induce endothelial cell inflammation. J Lipid Res 50: 204-213.

23. den Hartigh LJ, Connolly-Rohrbach JE, Fore S, Huser TR, Rutledge JC (2010) Fatty acids from very low-density lipoprotein lipolysis products induce lipid droplet accumulation in human monocytes. J Immunol 184: 3927-3936.

24. Schippling S, Kontush A, Arlt S, Buhmann C, Stürenburg HJ, et al. (2000) Increased lipoprotein oxidation in Alzheimer's disease. Free Radic Biol Med 28: 351-360.

25. Shishehbor MH, Zhang R, Medina H, Brennan ML, Brennan DM, et al. (2006) Systemic elevations of free radical oxidation products of arachidonic acid are associated with angiographic evidence of coronary artery disease. Free Radic Biol Med 41: 1678-1683.

26. Eiserich JP, Yang J, Morrissey BM, Hammock BD, Cross CE (2012) Omics approaches in cystic fibrosis research: a focus on oxylipin profiling in airway secretions. Ann N Y Acad Sci 1259: 1-9.

27. Folstein MF, Folstein SE, McHugh PR (1975) "Mini-mental state". A practical method for grading the cognitive state of patients for the clinician. J Psychiatr Res 12: 189-198

28. Friedewald WT, Levy RI, Fredrickson DS (1972) Estimation of the concentration of low-density lipoprotein cholesterol in plasma, without use of the preparative ultracentrifuge. Clin Chem 18: 499-502.

29. Shearer GC, Newman JW (2008) Lipoprotein lipase releases esterified oxylipins from very low-density lipoproteins. Prostaglandins Leukot Essent Fatty Acids 79: 215-222.

30. Keenan AH, Pedersen TL, Fillaus K, Larson MK, Shearer GC, et al (2012) Basal omega-3 fatty acid status affects fatty acid and oxylipin responses to high-dose n3-HUFA in healthy volunteers. J Lipid Res 53: 1662-1669.

31. Strassburg K, Huijbrechts AM, Kortekaas KA, Lindeman JH, Pedersen TL, et al. (2012) Quantitative profiling of oxylipins through comprehensive LC-MS/MS analysis: application in cardiac surgery. Anal Bioanal Chem 404: 1413-1426.

32. Fiehn O1, Kind T (2007) Metabolite profiling in blood plasma. Methods Mol Biol 358: 3-17.

33. Fiehn O, Wohlgemuth G, Scholz M, Ludäscher B, Raschid L (2005) Setup and Annotation of Metabolomic Experiments by Integrating Biological and Mass Spectrometric Metadata. Data Integration in the Life Sciences: Springer Berlin / Heidelberg. pp. 735-735.

34. Scholz M, Fiehn O (2007) SetupX--a public study design database for metabolomic projects. Pac Symp Biocomput .

35. Gilfix BM, Blank DW, Rosenblatt DS (1997) Novel reductant for determination of total plasma homocysteine. Clin Chem 43: 687-688.

36. Volek JS, Sharman MJ, Gomez AL, Scheett TP, Kraemer WJ (2003) An Isoenergetic Very Low Carbohydrate Diet Improves Serum HDL Cholesterol and Triacylglycerol Concentrations, the Total Cholesterol to HDL Cholesterol Ratio and Postprandial Lipemic Responses Compared with a Low Fat Diet in Normal Weight, Normolipidemic Women. J Nutr 133: 2756-2761.

37. Mallat Z, Nakamura T, Ohan J, Lesèche G, Tedgui A, et al. (1999) The relationship of hydroxyeicosatetraenoic acids and F2-isoprostanes to plaque instability in human carotid atherosclerosis. J Clin Invest 103: 421-427.

38. Clarke R, Smith A, Jobst KA, Refsum H, Sutton L, et al. (1998) Folate, vitamin b12, and serum total homocysteine levels in confirmed alzheimer disease. Archives of Neurology 55: 1449-1455. 
Citation: $\quad$ Altman R, Keenan AH, Newman JW, Rutledge JC (2014) The Postprandial Effects of a Moderately High-Fat Meal on Lipid Profiles and Vascular Inflammation in Alzheimer's Disease Patients: A Pilot Study. J Gen Practice 2: 186. doi:10.4172/2329-9126.1000186

Page 9 of 9

39. Miller JW (1999) Homocysteine and Alzheimer's disease. Nutr Rev 57: 126-129.

40. Regland B, Abrahamsson L, Gottfries CG, Magnus E (1990) Vitamin B12 Analogues, Homocysteine, Methylmalonic Acid, and Transcobalamins in the Study of Vitamin B12 Deficiency in Primary Degenerative Dementia. Dement Geriatr Cogn Disord 1: 272-277.

41. Akiyama H, Barger S, Barnum S, Bradt B, Bauer J, et al. (2000) Inflammation and Alzheimer's disease. Neurobiol Aging 21: 383-421.

42. Town T, Vendrame M, Patel A, Poetter D, DelleDonne A, et al. (2002) Reduced Th1 and enhanced Th2 immunity after immunization with Alzheimer's beta-amyloid(1-42). J Neuroimmunol 132: 49-59.

43. Xu M, Mizoguchi I, Morishima N, Chiba Y, Mizuguchi J, et al. (2010) Regulation of antitumor immune responses by the IL-12 family cytokines, IL-12, IL-23, and IL-27. Clin Dev Immunol 2010.

44. Rutz S, Ouyang W (2011) Regulation of interleukin-10 and interleukin-22 expression in $\mathrm{T}$ helper cells. Curr Opin Immunol 23: 605-612.

45. Schwarz MJ, Chiang S, Müller N, Ackenheil M (2001) T-helper-1 and Thelper-2 responses in psychiatric disorders. Brain Behav Immun 15 340-370.

46. Singh VK, Guthikonda P (1997) Circulating cytokines in Alzheimer's disease. J Psychiatr Res 31: 657-660.

47. Engelborghs S, De Brabander M, De Cree J, D'Hooge R, Geerts H, et al. (1999) Unchanged levels of interleukins, neopterin, interferon-gamma and tumor necrosis factor-alpha in cerebrospinal fluid of patients with dementia of the Alzheimer type. Neurochem Int 34: 523-530.

48. Rota E, Bellone G, Rocca P, Bergamasco B, Emanuelli G, et al. (2006) Increased intrathecal TGF-beta1, but not IL-12, IFN-gamma and IL-10 levels in Alzheimer's disease patients. Neurol Sci 27: 33-39.

49. Tarkowski E, Wallin A, Regland B, Blennow K, Tarkowski A (2001) Local and systemic GM-CSF increase in Alzheimer's disease and vascular dementia. Acta Neurol Scand 103: 166-174.
50. Romas SN, Tang MX, Berglund L, Mayeux R (1999) APOE genotype, plasma lipids, lipoproteins, and AD in community elderly. Neurology 53: 517-521.

51. Cunnane SC, Schneider JA, Tangney C, Tremblay-Mercier J, Fortier M, et al. (2012) Plasma and brain fatty acid profiles in mild cognitive impairment and Alzheimer's disease. J Alzheimers Dis 29: 691-697.

52. Guan Z, Wang Y, Cairns NJ, Lantos PL, Dallner G, et al. (1999) Decrease and structural modifications of phosphatidylethanolamine plasmalogen in the brain with Alzheimer disease. J Neuropathol Exp Neurol 58: 740-747.

53. Prasad MR, Lovell MA, Yatin M, Dhillon H, Markesbery WR (1998) Regional membrane phospholipid alterations in Alzheimer's disease. Neurochem Res 23: 81-88.

54. Miller JW (2000) Homocysteine, Alzheimer's disease, and cognitive function. Nutrition 16: 675-677.

55. Aljada A, Mohanty P, Ghanim H, Abdo T, Tripathy D, et al. (2004) Increase in intranuclear nuclear factor kappaB and decrease in inhibitor kappaB in mononuclear cells after a mixed meal: evidence for a proinflammatory effect. Am J Clin Nutr 79: 682-690.

56. Bellido C, Lopez-Miranda J, Blanco-Colio LM, Perez-Martinez P, Muriana FJ, et al. (2004) Butter and walnuts, but not olive oil, elicit postprandial activation of nuclear transcription factor kappaB in peripheral blood mononuclear cells from healthy men. Am J Clin Nutr 80: 1487-1491

57. Carroll MF, Schade DS (2003) Timing of antioxidant vitamin ingestion alters postprandial proatherogenic serum markers. Circulation 108 24-31.

58. Peairs AD, Rankin JW, Lee YW (2011) Effects of acute ingestion of different fats on oxidative stress and inflammation in overweight and obese adults. Nutr J 10: 122. 